\title{
Jóvenes catalanes en Londres: emigración juvenil, crisis y mercado de trabajo
}

\section{Young Catalan people in London: youth emigration, crisis and labour market}

\author{
Clara Rubio Ros*
}

Universitat Autónoma de Barcelona, Barcelona, España http://orcid.org/o0oo-000I-5892-5805

ISSN: ISSN-OI85-4259; e-ISSN: 2007-9I76

DoI: http://dx.doi.org/I0.28928/revistaiztapalapa/852018/aot2/rubiorosc

\section{Resumen}

Debido al contexto de crisis económica en España, muchas personas jóvenes ven difícil dar salida a su carrera profesional y deciden hacerlo en otro país; otros toman la crisis como una oportunidad para irse al extranjero y tener una experiencia internacional. Londres es uno de los destinos preferidos, entre otros motivos, por la oportunidad de aprender inglés, de encontrar trabajo y por su proximidad con España.

Este artículo contextualiza el estado de la juventud en España en términos laborales, así como el proceso migratorio que desde 2008 están llevando a cabo muchos jóvenes españoles hacia el extranjero, poniendo especial énfasis en el Reino Unido. A partir del estudio de caso de treinta jóvenes catalanes se pretende dar voz a las experiencias y reflexiones de los jóvenes emigrantes, mostrando sus estrategias de inserción laboral y sus trayectorias laborales en Londres. Palabras clave: precariedad laboral, migración, España, Reino Unido

\footnotetext{
Abstract:

Many young people are having dificulties to start their profesional career in Spain due to the current economical crisis, and decide to move abroad, others just consider the economic crisis an opportunity to go abroad and have an international experience. London is one of the prefered destinations because of different reasons: it provides a good opportunity to learn English, the country has ample job opportunities and it is close to Spain.

This paper approaches the state of youth in Spain in terms of employment and the migration process adopted by many Spaniards since 2008 , focusing specifically in the $\mathbf{U K}$ as destination.

This paper aims to give voice to the 30 young Catalan people interviewed, showing their migration and labour strategies in London. Keywords:, labour precariousness, migration, Spain, United Kingdom
}

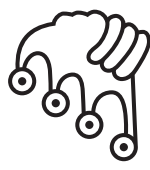

IZTAPALAPA

Agua sobre lajas

* clara.ruro@gmail.com 


\section{Introducción}

esde los inicios de los estudios de migración se han relacionado los procesos
migratorios con una voluntad de los migrantes de mejorar su situación
económica, social y personal. Este artículo trata de un colectivo específico, la juventud española, que debido a la fuerte desindustrialización sufrida en España en los años ochenta, así como a la crisis económica en la que se encuentra el país desde 2008 y las consecuentes altas tasas de desempleo y de precariedad laboral, ha empezado a emigrar a otros países en busca de mejores condiciones de empleo. Este fenómeno ha cambiado el curso de los últimos años, de tal forma que España ha pasado de ser un país de inmigración en los años 2000 para volver a ser un país de emigración en 2013 , como lo fue en los años sesenta. No obstante, el perfil del emigrado ha cambiado desde entonces, mientras que en aquella época salían al extranjero jóvenes poco calificados y en contexto de dictadura, ahora lo hacen mayoritariamente personas jóvenes con titulaciones universitarias y en un contexto de democracia política (Feixa y Rubio, 2017).

De manera estructural, la juventud está en clara desventaja para encontrar trabajo en las sociedades actuales, en especial en mercados laborales más rígidos (Fashoyin, 20I2). En el caso de España esta dificultad ha sido particularmente importante desde los años ochenta, cuando el país sufrió una fuerte desindustralización y apostó por sectores como el turismo, la hotelería y la construcción como bases de la economía, desarrollando así un tejido productivo dependiente de sectores con bajo valor añadido, con poca inversión en investigación y desarrollo y sin posicionamiento industrial, elementos que han ayudado a normalizar la precariedad (Petras, 1995). No obstante, esta situación ha empeorado debido a la reciente crisis económica, llegando a una tasa de desocupación juvenil (I5-29 años) de 42.2\% en 2013, cifra que ha ido disminuyendo paulatinamente hasta llegar a 39.7\% en 2015. Más allá del desempleo, las personas jóvenes se encuentran ante condiciones laborales inestables y trabajos para los que estan sobrecalificados (Serracant, 2005; Salvadó, 2009; Barbagelata, 2012). Aunque es común que la juventud tenga un periodo de desempleo 
al finalizar los estudios y que los primeros trabajos a los que acceden tiendan a ser temporales; la investigación hecha por Scarpetta et al. (2010) muestra que el primer empleo tiene importancia en la trayectoria profesional futura. El mismo estudio muestra que entre 1995 y 2005 la tasa de personas jóvenes en puestos laborales inestables aumentó $1.5 \%$, siendo España uno de los países donde la juventud pasaba más tiempo parada o en trabajos precarios antes de conseguir uno que correspondiera con su formación. Los estudios académicos apuntan a que en el contexto de crisis actual es muy probable que esta tasa sea mayor. En este sentido, Salvadó (2009), Serracant (2005) y Bessant et al. (2017) opinan que la precariedad laboral es un factor estructural que afecta a toda una generación, que arrastrará consigo trabajos temporales e inestables toda su vida, y no un factor coyuntural de la edad, como apuntan estudios hechos por la OCDE.

En este contexto, no sorprende que la juventud perciba pocas oportunidades laborales en su propio país y la emigración surja como salida para muchos de ellos. Según González-Ferrer (2013), el Reino Unido es el principal destino de los emigrantes españoles según las inscripciones consulares, seguido por Francia y por Alemania. En este artículo proponemos aproximarnos a ese fenómeno por medio del estudio de caso de la juventud catalana calificada en Londres. Se trata de un fenómeno que en los inicios de la crisis económica el gobierno español consideró como "aventura", catalogándolo como algo positivo para el país; pero que algunos movimientos sociales como Juventud sin Futuro o Marea Granate lo han asumido como una expulsión y así lo han puesto de manifesto en sus diferentes movilizaciones. Siguiendo el argumento de estos movimientos sociales, algunos estudios muestran cómo la juventud española ha integrado el argumento de la falta de opciones laborales en su país como uno de los motivos principales por los que se fueron (Rubio y Strecker, 20I6; Rubio y Strecker, 2017). Así pues, afirman marcharse porque no encuentran trabajo en España pero, ¿qué es lo que estos jóvenes encuentran en el país de acogida?, ¿consiguen un empleo calificado? ¿cómo lo hacen? El presente artículo pretende, por medio de un trabajo etnográfico, conocer las experiencias de la juventud calificada catalana $^{1}$ en el mundo laboral de Londres, dando voz a las historias y reflexiones que hacen sobre el proceso migratorio. Se escogió el colectivo catalán por razones prácticas, siendo este el colectivo al que la autora tenía acceso, y Londres por el imaginario colectivo que esta ciudad tiene en España, siendo considerada una urbe cosmopolita con muchas oportunidades socioculturales y laborales.

1 En este artículo se entiende como juventud calificada aquella con estudios universitarios 
El artículo está organizado en cinco apartados. El primero es una revisión de la literatura en los temas de migración juvenil en el contexto socioeconómico español; el segundo aborda el fenómeno de la emigración haciendo un repaso de los datos estadísticos disponibles hasta el momento; el tercero recoge la metodología utilizada en la investigación; el cuarto muestra los resultados del trabajo de campo en materia de ocupación y empleo entre las personas jóvenes catalanas entrevistadas. Finalmente, el artículo expone algunas conclusiones que relacionan la literatura trabajada con los resultados encontrados en el trabajo de campo.

\section{Estado del bienestar, capital y migración}

La crisis económica en España y los recortes presupuestarios han afectado de manera especial las políticas públicas. Una de las partidas más debilitadas ha sido la de juventud, al recortar muchos de los programas destinados a la igualdad de oportunidades entre las personas jóvenes en España. En este contexto, la familia ha pasado a sustituir las políticas públicas y se ha encargado de garantizar el bienestar de las personas jóvenes y facilitar su transición a la vida adulta (Walther, 2006).

Ante la falta de oportunidades laborales para este sector de la población, las experiencias internacionales pasan a ser una estrategia viable para muchos de ellos. Vivir en el extranjero durante un periodo de tiempo les permite adquirir varias competencias: instrumentales -cómo comunicarse en un idioma extranjero-, técnicas -las propias de la profesión-, y transversales - tales como la autonomía o la flexibilidad- (Alberich et alt, 2016), las cuales les sería mucho más costoso adquirir si se quedaran en España. De este modo, ante la amenaza de permanecer en España y encontrarse con largas etapas de desempleo, los jóvenes deciden irse al extranjero para evitar un vacío temporal en sus currículos y a la vez desarrollar ciertas competencias que les servirán para su inserción laboral futura (Rubio y Strecker, 2017). No obstante, conviene remarcar que la crisis económica muchas veces acaba siendo un empujón para hacer algo que siempre les había llamado la atención, más que una situación de desesperación porque, como se verá en los resultados, muchos de ellos no habían ni intentado buscar trabajo en España antes de irse a Londres (Rubio, 20I7).

Además, al convertise la familia en el principal pilar de bienestar de las personas jóvenes, sus oportunidades también dependeran de las posibilidades familiares, incluso la opción de irse al extranjero. En este sentido es pertinente recordar la interpretación de Portes y Hoffman (2003), que ubica una fuerte relación entre 
migración y capital, y según la cual salir del país es mucho más fácil y viable para aquellas personas con un capital superior: los que pueden pagar el viaje (capital económico), que conocen alguien a destino (capital social) y que tienen un nivel educativo superior (capital cultural). El capital económico les permite costear el proceso migratorio; el capital social, tener $\mathrm{y} / \mathrm{o}$ establecer contactos que faciliten el proceso (Castles y Miller, 2003; Faist y Ozveren, 2004), y el capital cultural es el que les permite adaptarse más rápido y fácil a la sociedad de acogida (Kuptsch y Pang, 2006). Este apunte es especialmente significativo en el contexto español en el que, a causa de la debilitación del Estado de bienestar y la reducción de políticas públicas en materia de juventud, la gente joven ha dependido más de los recursos familiares para poder llevar a cabo su transición a la vida adulta así como en el proceso migratorio, el cual ha llegado a ser más factible para jóvenes con famílias de clases superiores. Se podría pensar que estas diferencias hubieran disminuido gracias a las facilidades tecnológicas y de transporte que existen, pero no ha sido así y continúan siendo importantes (Jiménez, 20II).

En referencia a la relación entre el capital y la migración viene a cuento la aportación de Favell et al. (2006) y su crítica a la falsa polarización que se ha hecho desde los estudios sobre el tema, al considerar a los migrantes como elite o como proletariado sin discriminar la existencia de una gran parte que no se ubican en ninguna de las categorías extremas, lo que el autor llama "middle migrants", colectivo al que se ascriben los informantes de esta investigación:

[Más allá de] las elites globales que tienen acceso a vuelos frecuentes y a carriles rápidos [los cuales son más comunes] en revistas de actualidad y folletos corporativos, hay otras realidades sociales diferenciadas: estudiantes, enfermeras, técnicos medios y clérigos; gente de clase media ambiciosa y con ganas de aventura, migrantes procedentes de diferentes estados en desarrollo entre otros (Favell et al., 2006: 2).

De este modo, pues, las voces y experiencias que se recogen provienen de los "middle migrants", jóvenes que tienen estudios universitarios, que provienen de clases medias y que consideran la emigración como una oportunidad para enriquecer su currículo y aumentar sus posibilidades de encontrar empleo. 


\section{Emigración juvenil española en números}

El Barómetro del Centro de Investigaciones Sociológicas de febrero de 2012 muestra cómo $48 \%$ del total de la población española estaba dispuesta a irse al extranjero. Las cifras han ido aumentando con el tiempo. Un estudio hecho por el Cambridge Monitor en 2015 muestra que $84 \%$ de los milenial españoles piensa que tendrá que irse al extranjero en un futuro, mientras que un trabajo Rubio y Strecker en 2017 muestra cómo 90\% de los universitarios catalanes piensan tener una experiencia internacional después de concluir sus estudios. Esta diferencia puede ser explicada por el análisis de González-Ferrer (2013), quien sugiere que el nivel de preparación académica es importante para entender los planes de emigración de los jóvenes: aquellos con estudios superiores quieren irse en mayor grado que quienes no los tienen, dato también mostrado en el informe del Barómetro del Real Instituto Elcano del febrero de 2013 , donde se expone que $35 \%$ de la juventud española con estudios primarios piensa en irse al extranjero, mientras que la cifra sube a $65 \%$ para los que poseen estudios universitarios.

En este sentido es muy pertiniente atender lo que Domingo y Sabater bautizaron en 2013 como "profecía autocompleta": que la juventud española, ante las altas tasas de desempleo juvenil y un futuro cada vez más incierto, acaba creyendo que su única opción para no caer en lo que los medios de comunicación llamaron "generación perdida" es, precisamente, emigrar (Domingo y Sabater, 2013). Así, la emigración acaba siendo una opción que se va extendiendo cada vez más entre la juventud española calificada, dando lugar a un "efecto contagio" en el que mudarse al extranjero "con o sin proyecto, con o sin posibilidades reales, se acaba imponiendo como recurso" (Domingo y Blanes, 2015). Este elemento influye en que los y las jóvenes universitarias se lo planteen como una de las únicas opciones que tienen, incluso antes de intentar buscar trabajo en el país, para no caer en el desempleo tras acabar sus estudios,.

Pero ¿`cuántos jóvenes realmente se han ido? Según el Padrón de Españoles Residentes en el Extranjero (PERE), el número acumulado de personas jóvenes españolas entre 20 y 29 años que han emigrado al extranjero ha subido $41 \%$ entre 2008 y 2012 , pasando de 5538 a 7808 jóvenes emigrados. No obstante, estos datos no son totalmente fiables si se tiene en cuenta que dentro de la Unión Europea (que es donde más jóvenes españoles han emigrado durante estos últimos años) no es necesario darse de alta en el consulado español para poder trabajar en otros Estados miembros. De manera que es importante contrastar las estadísticas de migración con los datos de llegada de los países receptores para obtener el número más fiable posible 
de jóvenes españoles en el extranjero. El análisis de González-Ferrer (2013) explica la gran diferencia entre los datos disponibles en España y los del país de acogida: mientras el gobierno español calcula, en diferentes fuentes del Instituto Nacional de Estadística (EVR, е Рова, Estadística de Migraciones), que hay un total de 225000 españoles en el extranjero, los países receptores muestran una cifra que asciende a 700000 personas, llegando a ser más de tres veces superior. La autora estima que esta diferencia podría llegar a ser entre cuatro y siete veces superior en el caso del Reino Unido y Alemania, dos de los países con más inmigración de españoles.

En el caso del Reino Unido el registro más idóneo es el NiN (National Insurance Number), que recoge las peticiones del número de seguridad social hechas por parte de la población inmigrada. Si bien este registro tiene dos limitaciones importantes: no incluye a todas las personas ingresadas al Reino Unido (especialmente a aquellos que no solicitan el NIN por ser estudiantes o voluntarios, o quienes llegan al Reino Unido habiendo obtenido el NIN en migraciones pasadas) y tampoco tiene en cuenta a las personas que se marchan del país, ya que solo registra las entradas. Este es el conteo disponible más fiable.

La gráfica I muestra el número total de inscripciones de personas jóvenes entre I8 y 29 años en el sistema de seguridad social británico desde los inicios de la crisis económica; evidencia el gran aumento de la población juvenil española en el Reino Unido, al informar que en 2015 fue 3.7 veces mayor que en 2008 .

\section{Gráfica I.}

Registro del National Insurance Number de personas jóvenes entre 18 y 29 años, 2008 a 2015

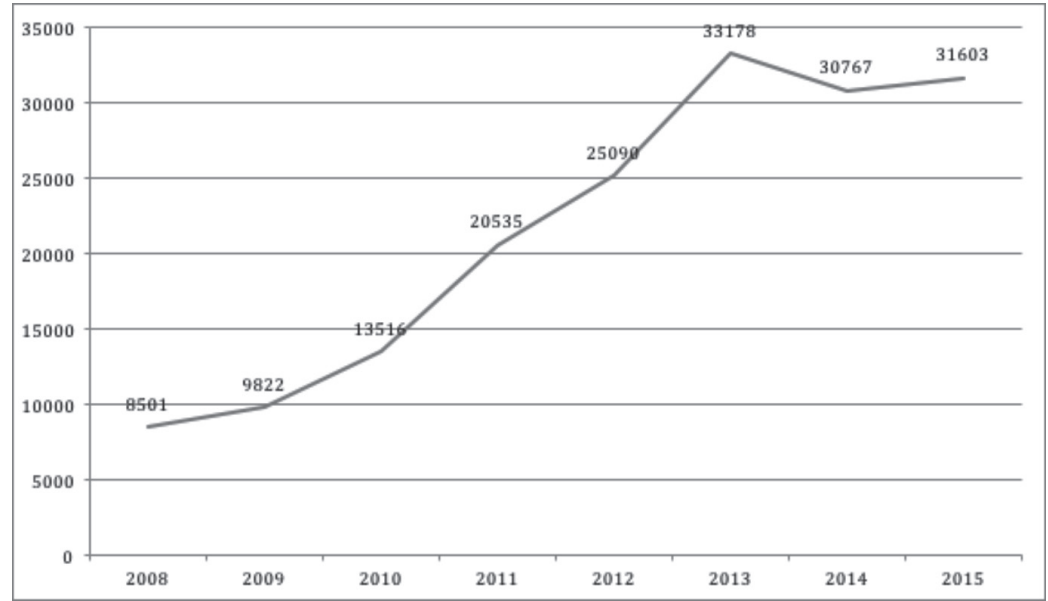

Fuente: National Statistics 


\section{Inmigración en Reino Unido}

La literatura registra como las motivaciones más comunes entre los emigrantes para irse de su país es la búsqueda de mejor calidad de vida (Kalter, 1998) y mejores condiciones en términos económicos (Hatton y Williamson, 1994). Pero en el caso de la juventud catalana emigrada a Londres ¿̇ qué influyó en su decisión de irse?

En la obra de Hooghe et al. (2008) se pone de manifiesto que, en términos generales, los procesos migratorios se pueden explicar desde tres puntos de vista. El primero, y el más antiguo, es el económico, que relaciona los procesos de migración con un ajuste de los mercados laborales entre países. El segundo, el cultural, explica los procesos de migración a partir de la relación centro-periferia que se dan por motivos de dominio lingüístico o de hegemonía cultural, causada principalmente por procesos históricos de colonización. Finalmente, el de las redes sociales, según el cual se aprovechan relaciones sociales previamente establecidas entre los migrantes.

Siguiendo el estudio de Hooghe et al. (2008), la explicación económica es la que ha tenido más impacto entre la juventud catalana emigrada a Londres. Mientras en España las tasas de paro son significativamente más elevadas que la media de la OCDE, el Reino Unido requiere trabajadores para poder ocupar vacantes. El estudio de la Fundación CIPD (2013) explica que los sitios de trabajo en el Reino Unido han crecido de 28.I millones en 2002 a 29.8 millones en 20I2. Como consecuencia a este aumento significativo en oportunidades laborales, en el Reino Unido también han aumentado las personas inmigrantes en $60 \%$ desde 2002 hasta $2012: 17 \%$ de la mano de obra ocupada no nació en el Reino Unido. Las tasas de empleo más altas entre personas no nacidas en el Reino Unido son: 79.9\% sudafricanos, 79.94\% miembros de la Unión Europea y 78.7\% nacidos en Australia y Nueva Zelanda. En referencia al tiempo que tardan en conseguir un trabajo tras graduarse, la transición es más rápida en el Reino Unido que en España: en el año 2000, la juventud inglesa tardaba 19.4 meses para encontrar cualquier trabajo y 36.I meses para obtener uno permanente; mientras que en España se pasaban 34.6 meses para conseguir cualquier empleo y 56.6 meses para hallar uno permanente (Scarpetta et al., 2010). Además, existe un estereotipo construido por los medios de comunicación sobre el Reino Unido como "paraíso" para la juventud más calificada, que lo pinta como un país con grandes oportunidades para esa población (Rodríguez-Puertas y Entrena-Durán, 2017)

También se ha detectado una influencia cultural en la decisión de emigrar a Londres. El interés de las personas jóvenes españolas de vivir y trabajar en el Reino Unido puede ser explicado por un motivo de hegemonía cultural. Actualmente, y aún más desde que empezaron los procesos de globalización, la lengua inglesa se ha con- 
vertido en vehículo de los negocios y las relaciones internacionales, siendo evidente su importancia en muchos de los sitios de trabajo calificado. España actualmente es uno de los países con un nivel de inglés más bajo (PISA, 2012) y la juventud percibe la necesidad de mejorar sus competencias en esa lengua para aspirar a un trabajo acorde con sus expectativas laborales. Entre los diferentes países de habla inglesa a los que pueden emigrar, el Reino Unido se escoge por su proximidad geográfica con España y por las oportunidades socioculturales y laborales que ofrece.

\section{Metodología}

Durante el trabajo de campo en Londres, entre diciembre de 2012 y noviembre de 2015, se aplicaron cuatro diferentes métodos de acopio de información: entrevistas en profundidad, grupos focales, observación participante y etnografía. Todos los datos obtenidos se analizaron siguiendo la teoría fundamentada. Es importante tener en cuenta que esta investigación se llevó a cabo antes del Brexit, y que las opiniones de los informantes podrían ser diferentes en un contexto posterior.

Se hicieron treinta entrevistas en profundidad a un grupo homogéneo de jóvenes catalanes calificados emigrados a Londres, los cuales respondían a diferentes variables: edad, sexo, tiempo que llevan viviendo ahí, rama de sus estudios y experiencia laboral previa. A continuación se muestra una tabla resumen del perfil de los informantes:

\begin{tabular}{|c|c|c|c|c|c|c|}
\hline Nombre & Sexo & Edad & Llegada a Londres & Estudios & $\begin{array}{l}\text { Experien- } \\
\text { cia previa } \\
\text { en } U K^{1}\end{array}$ & $\begin{array}{l}\text { Fecha } \\
\text { entrevista } \\
(d d / m m / a a)\end{array}$ \\
\hline Clàudia & M & 23 & Enero 2012 (con pareja) & Periodismo & $\mathrm{B}$ & $\mathrm{I} 4-\mathrm{OI}-\mathrm{I} 3$ \\
\hline Lluís & $\mathrm{H}$ & 23 & Enero 2012 (con pareja) & Periodismo & $\mathrm{B}$ & $\mathrm{I} 4-\mathrm{OI}-\mathrm{I} 3$ \\
\hline Roger & $\mathrm{H}$ & 27 & Enero 2012 (con pareja) & Arquitectura & $\mathrm{TC}$ & $20-\mathrm{OI}-\mathrm{I} 3$ \\
\hline Laia & M & 27 & Octubre 2009 (sola) & Física médica & $\mathrm{E}$ & $24-\mathrm{O} 3-\mathrm{I} 3$ \\
\hline Marta & $\mathrm{M}$ & 25 & $\begin{array}{l}\text { Octubre } 2009 \text { (con } \\
\text { pareja) }\end{array}$ & Infermería & $\mathrm{E}$ & $3 \mathrm{I}-\mathrm{OI}-\mathrm{I} 3$ \\
\hline David & $\mathrm{H}$ & 22 & Septiembre 2009 (solo) & Lingüística & $\mathrm{E}$ & IO-I2-I2 \\
\hline
\end{tabular}




\begin{tabular}{|c|c|c|c|c|c|c|}
\hline Sara & M & 29 & Enero 2010 (con pareja) & $\begin{array}{l}\text { Periodismo } \\
\text { Humanida- } \\
\text { des }\end{array}$ & TNC & $2 \mathrm{I}-\mathrm{O} 3-\mathrm{I} 3$ \\
\hline $\begin{array}{l}\text { Gus- } \\
\text { tavo }\end{array}$ & $\mathrm{H}$ & 30 & Enero 2010 (con pareja) & $\begin{array}{l}\text { Humanida- } \\
\text { des } \\
\text { Fotografía }\end{array}$ & TC & $30-\mathrm{OI}-\mathrm{I} 3$ \\
\hline Xènia & M & 26 & Marzo 2012 (sola) & Estilismo & TC & $\mathrm{II}-\mathrm{I} 2-\mathrm{I} 2$ \\
\hline Marc & $\mathrm{H}$ & 25 & Septiembre 20II (solo) & Marqueting & $\mathrm{TC}$ & $\mathrm{II}-\mathrm{I} 2-\mathrm{I} 3$ \\
\hline Clara & M & 29 & $\begin{array}{l}\text { Noviembre } 2010 \text { (con } \\
\text { pareja) }\end{array}$ & Pedagogia & TC & $3 \mathrm{I}-\mathrm{OI}-\mathrm{I} 4$ \\
\hline $\mathrm{J}$ & $\mathrm{H}$ & 29 & $\begin{array}{l}\text { Noviembre } 2010 \text { (con } \\
\text { pareja) }\end{array}$ & Ingeniería & TC & $\mathrm{O} 8-\mathrm{O} 2-\mathrm{I} 4$ \\
\hline Max & $\mathrm{H}$ & 24 & Junio 2013 (solo) & Periodismo & TC & $30-\mathrm{OI}-\mathrm{I} 4$ \\
\hline Joan & $\mathrm{H}$ & 27 & $\begin{array}{l}\text { Febrero } 2014 \text { (con } \\
\text { pareja) }\end{array}$ & Arquitectura & TNC & O4-03-I4 \\
\hline Àfrica & M & 28 & Febrero 2014 (sola) & Infermeria & TC & $\mathrm{OI}-03-\mathrm{I} 4$ \\
\hline Andreu & $\mathrm{H}$ & 27 & Octubre 2013 (solo) & $\begin{array}{l}\text { Telecomuni- } \\
\text { ca-ciones }\end{array}$ & TNC & $24-\mathrm{O} 2-\mathrm{I} 4$ \\
\hline $\mathrm{M} \& \mathrm{M}$ & $\mathrm{H}$ & 32 & Octubre 2013 (solo) & Ingeniería & TNC & $24-\mathrm{O} 2-\mathrm{I} 4$ \\
\hline Martí & $\mathrm{H}$ & 23 & Octubre 2013 (solo) & $\begin{array}{l}\text { Telecomuni- } \\
\text { ca-ciones }\end{array}$ & TC & $06-12-13$ \\
\hline Blanca & M & 27 & Octubre 2013 (sola) & $\begin{array}{l}\text { Arquitectura } \\
\text { técnica }\end{array}$ & TNC & $20-\mathrm{OI}-\mathrm{I} 4$ \\
\hline Sergi & $\mathrm{H}$ & 25 & Febrero 2014 (solo) & $\begin{array}{l}\text { Adminis- } \\
\text { tración } \\
\text { Empresas }\end{array}$ & TNC & $\mathrm{I} 2-\mathrm{O} 3-\mathrm{I} 4$ \\
\hline $\begin{array}{l}\text { Mar- } \\
\text { tista }\end{array}$ & M & 26 & Febrero 2014 (sola) & Bellas artes & TNC & $13-03-14$ \\
\hline Júlia & M & 27 & Septiembre 2013 (sola) & Infermería & $\mathrm{TC}$ & $17-03-14$ \\
\hline Charlie & $\mathrm{H}$ & 24 & Marzo 2014 (solo) & $\begin{array}{l}\text { Diseño grá- } \\
\text { fico }\end{array}$ & $\begin{array}{l}\text { Desem- } \\
\text { pleado }\end{array}$ & $27-03-14$ \\
\hline Marçal & $\mathrm{H}$ & 25 & Febrero 2014 (solo) & Ingeniería & $\mathrm{TC}$ & $25-02-I 4$ \\
\hline
\end{tabular}




\begin{tabular}{|c|c|c|c|c|c|c|}
\hline Aina & M & 29 & Enero 2014 (sola) & Periodismo & B & I4-OI-I4 \\
\hline Oriol & $\mathrm{H}$ & 28 & Enero 2014 (solo) & Periodismo & B & $28-\mathrm{OI}-\mathrm{I} 4$ \\
\hline Rosa & $\mathrm{M}$ & 25 & Febrero 2014 (sola) & Periodismo & $\mathrm{B}$ & $04-03-14$ \\
\hline Sílvia & M & 22 & Febrero 2014 (sola) & Ingeniería & B & $\mathrm{IO}-03-\mathrm{I} 4$ \\
\hline $\begin{array}{l}\text { Geor- } \\
\text { gina }\end{array}$ & $\mathrm{M}$ & 38 & Septiembre 2014 (sola) & Magisterio & $\mathrm{TC}$ & $\mathrm{O} 2-\mathrm{O} 3-\mathrm{I} 5$ \\
\hline Míriam & M & 26 & Febrero 2015 (sola) & Derecho & TNC & $07-03-15$ \\
\hline
\end{tabular}

1 TC: Trabajo calificado; TNC: Trabajo no calificado; Eः Estudios; Bः Beca

Las entrevistas semipautadas se efectuaron presencialmente en la ciudad de Londres, con una duración media de 75 minutos. Las preguntas se estructuraron a partir de los cinco momentos de migración: la construcción de la idea de emigrar, el viaje hacia Londres, las condiciones de vida en esa ciudad, la visión que se tiene del propio país desde Londres y las perspectivas de futuro. Además, después de la entrevista en profundidad y hasta la finalización del trabajo de campo se hizo un seguimiento mensual de los informantes para conocer los posibles cambios personales y profesionales envueltos en el proceso migratorio. Posteriormente a esta entrevista se hizo un estudio longitudinal de entre tres meses y un año (dependiendo del primer contacto con cada uno de los jóvenes) en el que se recogieron los diferentes cambios laborales. A partir de estos datos recogidos en campo se han podido detectar diferentes trayectorias laborales de la juventud catalana emigrada a Londres, dependiendo de su recorrido para conseguir un trabajo calificado acorde con sus titulaciones. Es importante remarcar que todos ellos tenían planes temporales de emigración, pensaban volver a Cataluña en un futuro próximo, muchos marcaban la fecha de retorno condicionada a la mejora de la economía en España y otros al cumplimiento de sus objetivos iniciales (ya fuera aprender inglés o conseguir un empleo calificado en el mercado laboral británico).

Paralelamente, también se crearon tres grupos focales de las personas que ya habían participado en las entrevistas, con un total de ocho participantes en cada grupo. Estos grupos focales tenían el objetivo de discutir diferentes temáticas de interés para conocer el proceso migratorio, como las oportunidades laborales en Londres, las expectativas de empleo y las diferentes estrategias para conseguir colocación en la ciudad. 
De manera transversal, durante el trabajo de campo se hizo observación participante en las diferentes reuniones culturales y sociales que los individuos han hecho en Londres, organizadas por los propios emigrantes catalanes en la ciudad (como la fiesta de Sant Jordi ${ }^{2}$ o la celebración de Navidad); pero también se practicó observación participante en los diferentes grupos de facebook que catalanes y españoles emigrados a Londres han creado (Españoles en Londres, Catalans a UK, Catalans a Londres). En los dos casos de observación participante (presencial o virtual) se ha mostrando especial interés en los temas relacionados con los mercados de trabajo y la inserción laboral de la juventud catalana en Londres.

A continuación vamos a exponer los resultados del trabajo de campo. ${ }^{3}$ Están organizados en cuatro partes. En la primera aparecen las expectativas y las motivaciones para ir a vivir a la capital británica. En la segunda, cuál es el trabajo que estaban desarrollando en Londres en el momento de la entrevista, considerando cuatro variables y su impacto en su inserción laboral: su nivel de inglés, la rama del conocimiento de sus estudios, la experiencia profesional previa y el tiempo que llevaban viviendo en la ciudad. Al final de esta segunda parte también se combinan las variables "tiempo en la ciudad" con la "rama de conocimiento de sus estudios", dos variables explicativas de los procesos de inserción laboral en Londres. La tercera parte reportaba los seis diferentes perfiles de inserción que han seguido para entrar en el mercado laboral. La cuarta parte muestra una iniciativa de emprendimiento empresarial en torno a la emigración de jóvenes catalanes y españoles en Londres: las agencias de empleo.

Según los datos recogidos, todas las personas consultadas aspiran a conseguir en algún momento de su estancia en Londres un trabajo relacionado con sus estudios. No obstante, en el momento de la entrevista no todos estaban colocados en su empleo ideal, ${ }^{4}$ lo cual sugiere que la inserción de la juventud catalana en el mercado laboral de Londres responde a diferentes variables.

\section{Aspiraciones laborales en Londres}

2 La fiesta de San Jordi, patrón de Cataluña, es una festividad cuya tradición consiste en un intercambio de libros y rosas, como muestra de cariño.

3 Las citas de los informantes están recogidas a partir de los pseudónimos que ellos mismos eligieron en el momento de la entrevista.

4 Se utiliza el término "trabajo ideal" para referirse a empleos calificados y en concordancia con la formación previa de los informantes 
En general, podemos decir que la juventud catalana emigrada a Londres busca mejorar su nivel de inglés (expresado por 70\% de los y las informantes) y tener una experiencia laboral relacionada con sus estudios (expresado por 60\%). Entre las otras motivaciones señaladas se mencionó: tener una experiencia internacional, estudiar en el Reino Unido o hacer prácticas profesionales.

Aunque el aprendizaje del inglés y la experiencia laboral eran las motivaciones más comunes entre ellos, algunos manifestaron que en el momento de elegir destino dudaron que Londres fuera la mejor ciudad para adquirir la lengua, pero finalmente se decidieron a hacerlo por las oportunidades laborales que brinda la capital británica. Es el caso de Charly, quien explica cómo la alta concentración de población hispanohablante hace que a menudo sea difícil para los recién llegados encontrar un espacio donde practicar el idioma. Algunos de sus conocidos ya le habían advertido de ello, pero acabó dediciendo ir a Londres por considerar que las opciones laborales allí eran significativamente superiores a cualquier otra parte del Reino Unido.

El principal motivo que me hizo decidirme por Londres es que aquí era más fácil conseguir un trabajo que en cualquier parte del Reino Unido, ¡ hay más oportunidades en esta ciudad! Pero realmente me gustaría ir a otro sitio porque... mis amigos ya me lo avisaron, me dijeron que no aprendería inglés en Londres aunque yo pensara que me podría distanciar del guetto de españoles... (Charly)

Además de la opción de encontrar trabajo no calificado de manera rápida y sencilla, también consideraron Londres como un buen destino porque ofrece múltiples opciones de trabajo calificado, el cual les ayudará a mejorar su currículo y ampliar sus opciones de obtener un buen empleo al volver a España. Si bien es cierto que no todos aspiraban a conseguirlo de manera inmediata, sí era una aspiración común a la que planeaban llegar tarde o temprano.

\section{Inserción laboral en Londres}

Para analizar el tipo de puesto que tenían en el momento de la entrevista se han creado tres categorías. En función de si el trabajo correspondía con su nivel de estudios, utilizamos los términos trabajo calificado, ${ }^{5}$ incluyendo también si esta-

5 Los trabajos calificados incluyen: empleos en empresas públicas o privadas, prácticas 
ban en una rama del conocimiento diferente a la de sus respectivos estudios; si era un trabajo para el cual la persona joven estaba sobrecalificada; o si se encontraba desempleada. $63.3 \%$ de los y las jóvenes tenían un trabajo de acuerdo con su nivel de estudios, aunque no en todos los casos estaban relacionados con la titulación adquirida. Uno de los treinta informantes tuvo que cambiar de profesión, y cuatro de los diecinueve informantes que tenían trabajos calificados lo consiguieron por medio de programas de inserción laboral, como las becas Leonardo de Vinci (dos personas) o de programas de prácticas laborales internacionales (dos personas). Por el contrario, siete jóvenes tenían trabajos no calificados (la mayoría en el sector de la hotelería) y cuatro (I3.3\% del total), estaban desempleados. Los datos aquí mencionados muestran cómo el desempleo continúa presente entre las personas jóvenes emigradas, aunque en menor grado que en España. Las becas Leonardo de Vinci y las prácticas internacionales son consideradas como maneras seguras de entrar en el mercado de trabajo londinense. Todas las personas entrevistadas que formaban parte de uno de estos programas consideraban estas experiencias como una manera de llegar a Londres con un trabajo seguro. Las personas del grupo de becas Leonardo de Vinci tomaban la beca como su primera experiencia profesional en Londres para obtener un trabajo calificado en un corto periodo. Sucedía lo mismo entre las personas del grupo de prácticas internacionales, aunque estos últimos eran los que mostraban más flexibilidad con respecto a conseguir un trabajo en cualquier otra ciudad del mundo:

La beca Leonardo era para nosotros la combinación perfecta: tener una beca y estar en Londres una temporada con trabajo. Así ya no nos teníamos que preocupar de eso, son tres meses de trabajo pagado y después ya... ¡a probar suerte! (Clàudia)

Al margen de la inserción laboral gracias a programas como las becas Leonardo de Vinci, la manera más común de solicitar trabajo entre jóvenes emigrantes en Londres es entregando en mano los currículos en las diferentes tiendas, restaurantes o bares del centro de la ciudad. Eso obedece a que esa es la costumbre en Cataluña. No obstante, explican que si buscaran un trabajo calificado, presentarían sus solicitudes

laborales que se integran en los planes de estudios de los carreras universitarias que se cursan en España, becas Leonardo de Vinci (programa subvencionado por la Unión Europea que tiene el propósito de facilitar la inserción laboral a jóvenes europeos recién titulados a partir de una experiencia laboral internacional en alguno de los Estados miembros) y trabajos por cambio de carrera profesional, es decir, trabajos calificados pero en una rama diferente a los estudios universitarios hechos previamente. 
por medio de las páginas web de las empresas, en lugar de entregarlos en mano. A continuación se discuten los diferentes trabajos que han encontrado en Londres relacionándolos con las variables que aparecen en los análisis de resultados de la inserción laboral de los y las jóvenes catalanas en Londres.

La tipología del trabajo al que estas personas jóvenes pueden aspirar está estrechamente relacionada con su nivel de inglés previo. No obstante, aunque sí hay una tendencia a obtener mejores puestos si se posee un mayor nivel de inglés, no siempre ocurre así. Los datos indican que cuatro de los diecinueve informantes con empleo calificado tenían un nivel bajo de inglés (inferior o igual al B2 del marco europeo), y entre los que tenían trabajos no calificados la proporción era de dos a siete, y entre los que estaban desempleados, tres de cuatro. Los datos muestran que aunque el nivel de inglés no sea determinante, puede influir en la obtención de trabajos calificados.

Mi nivel de inglés todavía es bastante bajo. Puedo mantener una conversación con pinzas. Entonces, cuando buscas trabajo eso te limita bastante. Cuando tienes una entrevista de trabajo y te preguntan cualquier cosa no puedes estar diez segundos pensando la respuesta... (Marc)

A su vez, los datos recogidos muestran que la tipología de estudios finalizados influye claramente en la inserción laboral, condicionada por la demanda del mercado. Mientras que los titulados en salud tenían todos un empleo acorde con sus estudios, los titulados en ciencias sociales enfrentaban más problemas para conseguir un trabajo calificado. Entre estos últimos, solo cinco de doce tenían un trabajo remunerado calificado, mientras que tres estaban en trabajos no calificados, uno había cambiado de sector y tres se hallaban en programas de inserción laboral.

En Navidad ya había hecho dos entrevistas a dos hospitales y uno me cogió enseguida. Me ponían presión para que dijera un sí seguro y al final, después de muchas indecisiones, lo cogí. Cuando obtuve en PIN ${ }^{6}$ firmé el contrato y vine. El hospital incluso me puso una casa para los primeros días (África)

Es importante remarcar que quienes tenían trabajos en salud, en concreto en enfermería, eran mujeres, y su principal motivación para ir a Londres era poder estudiar la especialidad en obstetricia. Habían llegado a esa ciudad para poderse especializar,

- El pin es un registro británico de los enfermeros que están trabajando para el sistema sanitario público 
ya que en España el proceso era mucho más complejo, además de tener la idea de que en el Reino Unido había una gran demanda de profesionales de la enfermería y que podrían encontrar colocación fácilmente.

Por otro lado, en las entrevistas se insistió en la importancia de tener experiencia laboral en Inglaterra para conseguir un trabajo calificado en el país, y en que previamente a la contratación en un nuevo puesto, el empleador comprueba las referencias emitidas en trabajos anteriores, con la cual es muy importante obtener buenas referencias para poderse abrir puertas en el mundo laboral. Sin embargo, los datos reflejan que la experiencia laboral previa en Inglaterra no es esencial para conseguir un trabajo calificado, aunque en la mayoría de los casos ayuda. Como vemos, es común que los jóvenes que tienen trabajo calificado cuenten con experiencia laboral previa en el Reino Unido, aunque no necesariamente. No obstante, ninguno de los desempleados tenía experiencia previa en el país, especialmente, como veremos a continuación, entre aquellos titulados que ejercían profesiones altamente demandadas en el Reino Unido. En este sentido la experiencia laboral no era la variable más influyente, aunque sí tenía cierta influencia.

Aquí para conseguir un trabajo tienes que mentir. No me gustaría, pero tienes que hacerlo. Si voy a tirar currículums a una tienda, pongo que tengo experiencias en tiendas. Claro, el hecho de no saber el idioma y además tener que mentir sobre algo que no sabes, ino es fácil! Luego salen mal las entrevistas, pero es la única manera [de conseguir un trabajo] (Charlie)

El tiempo que las personas jóvenes llevaban viviendo en la ciudad sí condicionaba el tipo de trabajo que tenían. Mientras más tiempo llevaban viviendo en Londres, más trabajos calificados habían conseguido. Esta relación se podría explicar por tener más experiencias laborales en el país, con la consecuencia de poseer más referencias laborales; pero también por tener mejor nivel de inglés, conocer mejor el sistema laboral del país y estar más integrados en la sociedad que los acoge. Entre los nueve que llevaban más de dos años en Londres, ocho tenían trabajos calificados, mientras que entre quienes llevaban menos de seis meses, cuatro ocupaban puestos calificados, cuatro estaban en programas de inserción y cinco en trabajos no calificados.

De ese modo, se puede colegir que las personas jóvenes con estudios en ciencias sociales necesitan tener cierta experiencia laboral en Reino Unido para acceder a un trabajo calificado. No obstante, en el caso de las ciencias de la salud es más fácil conseguirlo al poco tiempo de vivir en Londres o incluso venir con un trabajo contratado desde España. En el caso de las ingenierías también tienen una inserción laboral 
más rápida, aunque no tan inmediata como los profesionales de la salud. Cinco de los ocho titulados en ciencias sociales tardaron más de dos años en encontrar un empleo calificado, mientras que en el caso de los ingenieros fueron dos de cinco.

\section{Perfiles de inserción laboral en Londres}

A partir de la información recogida en el trabajo de campo se han podido detectar diferentes maneras de integrarse en el mercado laboral. A continuación se exponen las seis estrategias que han sido detectadas para lograrlo.

La trayectoria más común es la de aproximación sucesiva, es decir, conseguir un trabajo no calificado que funcione como puente para obtener uno calificado. Tal fue el caso de nueve de las treinta personas entrevistadas. Cinco de los nueve informantes encontraron un trabajo relacionado con sus estudios, mientras que dos seguían en empleos no calificados y otros dos estaban pensando volver a Cataluña en un futuro próximo.

En relación con el manejo del idioma, los inmigrantes lo veían como una dificultad para conseguir un trabajo en algo relacionado con sus estudios. Es por este motivo que decidían empezar por un trabajo no calificado (en bares, restaurantes, tiendas, pubs o hoteles) para mejorar así su manejo del inglés y sentirse más seguros para después buscar trabajos relacionados con su profesión.

Tenía opción de trabajar limpiado habitaciones o como asistente de cocina en el mismo hotel. Realmente son trabajos duros físicamente pero también psicológicamente. A veces da un poco de impotencia saber que has estudiado tanto para trabajar de eso, pero mi fluidez en inglés aún no es suficiente (Joan)

La segunda estrategia detectada fue la de un grupo de personas que consiguió un trabajo calificado desde Cataluña y emigró a Londres teniendo ya un puesto asegurado. En este grupo encontramos a todas las jóvenes enfermeras entrevistadas, las cuales enviaron sus currículos desde Cataluña, hicieron un primer contacto con los hospitales a través de videollamada para concretar una entrevista presencial en Londres y discutir los detalles del puesto. También es el caso de un ingeniero en telecomunicaciones y de un arquitecto, aunque representan dos casos únicos dentro del colectivo de jóvenes profesionales en el ámbito tecnológico que encontraron trabajo desde España. El resto de informantes decidieron esperarse a estar en Londres para buscar trabajo. 
Desde Cataluña hice solicitud de trabajo a tres empresas diferentes por internet. Dos de ellas me quisieron conocer y que hiciera una entrevista con ellos. Al final me decidí por la de Londres, por la ciudad, pero también por el proyecto, que me parecía más interesante (Martí)

Una tercera estrategia seguida es la de solo buscar y aceptar trabajos relacionados con sus estudios, sin importar el costo, con el objetivo de evitar un posible desclasamiento. Los y las jóvenes que seguían esta estrategia no querían pasar por trabajos no calificados antes, y todos ellos acabaron colocándose el trabajo que querían. Quienes lo consiguieron más rápidamente fueron aquellos que contaban con su propia empresa y trabajaban como freelance. Los dos jóvenes que acabaron contratados por una empresa les tomó más tiempo. Uno de ellos tardó ocho meses en conseguir el empleo que buscaba, mientras que el otro trabajó de manera voluntaria durante cuatro meses antes que le ofrecieran un puesto remunerado.

Me he negado a trabajar de cosas que no fueran fotografía. Me tenía que limitar a ganar dinero sólo haciendo esto porque si no, es muy difícil mantenerse en la industria (Gustavo).

Como hemos visto, aprender inglés era una de las motivaciones más comunes para ir a vivir a Londres. Así, una cuarta estrategia era la de tomar un curso de inglés para mejorar su nivel, adaptarse a la nueva cultura y analizar las opciones laborales. Fue una estrategia seguida por tres personas. Al finalizar el curso solo una de ellas decidió quedarse a la ciudad, las otras volvieron a Cataluña a probar suerte con un dominio del inglés superior al que tenían antes de su experiencia en Londres.

Primero vine a Londres a hacer un curso de inglés, estuve cuatro meses en una academia. Mi objectivo era aprender inglés y tener un poco de tiempo libre, pero también hacer contactos. Ya estuve enviando $\mathrm{Cv}$ a diferentes sitios, primero a trabajos calificados y después a no calificados. Hasta que conseguí el trabajo que estoy ahora en un bar. (M\&M)

La quinta estrategia detectada era la de obtener una beca Leonardo de Vinci para insertarse en el nuevo contexto sociocultural de manera segura. Cuatro de las personas entrevistadas estaban haciendo o habían hecho una estancia laboral en Londres con ese respaldo. Todas ellas eran periodistas. De la misma manera que quienes vinieron a Londres a hacer un curso de inglés, en su caso la beca significaba una 
primera aproximación al mercado laboral de Londres. Les permitía una experiencia que hacía valer sus referencias profesionales, mejorar su nivel de inglés y buscar trabajo de manera activa para cuando se les acabara el subsidio de la beca.

En el momento de escribir este artículo, dos de los jóvenes aún tenían la beca vigente y dos más ya habían terminado. Uno de ellos, después de conseguir un trabajo en un área totalmente diferente a la del periodismo, decidió cambiar de profesión. El otro, tras un periodo de trabajos no calificados, decidió volver a Barcelona a desempeñarse como profesor de inglés en escuelas de idiomas.

En la empresa en la que estoy haciendo las prácticas de Leonardo de Vinci tengo la oportunidad de hacer contactos en mi sector. Entonces aprovecharé al máximo los cinco meses de la beca para hacer contactos y buscar trabajo de lo mío (Aina)

Por último, una estrategia marginal entre los entrevistados consistió en ir a Londres por motivos de estudio. Algunos lo hacían como práctica laboral internacional propia del plan de estudios de sus respectivas carreras universitarias en España y otros para estudiar una carrera o máster en una universidad de Londres, pero siempre como alternativa a España. Las jóvenes de prácticas laborales habían escogido una estancia internacional con el objetivo de enriquecer su currículo y valorar sus opciones laborales en Londres. Los y las jóvenes que vinieron a Londres a estudiar un máster o una carrera universitaria lo hicieron porque eran carreras que no podían cursar en España o bien los estudios sujetos a procesos de selección muy competitivos en el país de origen (lingüística, máster en física médica y especialización en partería).

Las jóvenes que vinieron para realizar prácticas laborales aún trabajando en el momento de escribir el artículo. Los y las jóvenes que habían ido a estudiar una carrera o máster en Londres habían decidido quedarse a vivir en la ciudad y estaban trabajando en empleos calificados que consiguieron de manera inmediata después de acabar sus estudios o incluso paralela a sus cursos.

Yo quería trabajar de física médica. Para eso en España tienes que hacer unas oposiciones muy duras. Yo no veía el sentido en eso si lo comparabas con el sistema inglés, por ejemplo, que funciona en hacer un máster y presentarte a trabajos con un proceso de selección normal. Así que me informé del sistema inglés y vine a especializarme aquí (Laia) 


\section{El papel de las agencias de empleo}

El auge de la emigración juvenil catalana y española ha sido una oportunidad empresarial para muchos. En esta sección analizaremos uno en concreto: las agencias de empleo, las cuales ofrecen sus servicios a jóvenes emigrantes para ayudarlos en su llegada a Londres.

Dejar la comodidad de tu país para lanzarte a la aventura de vivir y trabajar en otra ciudad desconocida asusta a muchos de los jóvenes emigrantes. Ante esto, las agencias de empleo garantizan apoyo y asesoramiento en el proceso de emigración, ofreciendo múltiples servicios: desde vivienda, entrevistas de trabajo, trabajo asegurado, teléfono móvil, inscripción a la seguridad social, transporte, traducción del currículum vitae, dependiendo de las necesidades y los deseos de la persona.

Uno de los jóvenes entrevistados vino a vivir a Londres a través de una agencia de empleo en Cataluña, y en todo momento se mostró satisfecho con la decisión:

Yo vine con una agencia que me encontró trabajo aquí y bueno, al final me salió bien la jugada. Pagué entre Iooo y I200 euros pero vine ya con trabajo. Dejé mi trabajo un viernes y el lunes siguiente ya estaba trabajando. Hay muchas agencias que solo te buscan entrevistas de trabajo, no trabajo. Yo creo que no he perdido el dinero, mucha gente hace la inversión de venir aquí y buscar trabajo, yo ya viene directamente y empecé a trabajar (Joan)

No obstante, se han detectado diferentes fraudes y ya son muchos los catalanes y españoles en Londres que lo desaconsejan. Como contrapartida, diferentes blogs que hablan sobre cómo vivir en Londres y comentarios en grupos de facebook, como "Españoles en Londres", se han encargado de dar información suficiente a los interesados para que puedan conseguir de manera gratuita lo mismo que ofrecen las agencias, por ejemplo, recomiendan consultar la página web "diariodeunlondinense" o "trucoslondres.com".

\section{Conclusiones}

Siguiendo el artículo de Hooghe et al. (2008), que muestra las tres principales explicaciones de los procesos migratorios, en el caso de la juventud catalana emigrada a Londres detectamos claramente dos; por un lado, una motivación de mejorar las oportunidades laborales durante o a consecuencia de la estancia en Londres, la cual 
va ligada a una explicación económica. Siendo consicientes de la falta de oportunidades laborales en España, o animados por el "efecto contagio" de otras personas jóvenes que han emigrado (Domingo y Blanes, 2015), la juventud decide desplazarse a Londres para mejorar directa o indirectamente sus opciones de trabajo, ya sea consiguiendo ahí un puesto calificado que les permita empezar o dar continuidad a su carrera profesional, o adquiriendo competencias como un mejor nivel de inglés o experiencia en el mercado laboral que les permita ampliar sus opciones de trabajo al volver a España. También se ha podido detectar un gran interés por emigrar a Londres como ciudad. Esa intención se explica por las oportunidades laborales que, se supone, ofrece la ciudad, pero también por la ocasión de aprender y mejorar el idioma, motivación que está relacionada con la segunda explicación que Hooghe et al. (2008) mencionan en su artículo, en referencia a la hegemonía cultural.

Más allá del discurso teórico sobre la emigración actual de la juventud española, hemos querido dibujar las condiciones de trabajo de estos migrantes en la capital inglesa. Para ello analizamos el impacto de diferentes variables que han aparecido como relevantes en los resultados del proceso de encontrar trabajo en una ciudad extranjera a partir de los casos estudiados. De esta manera, concluimos que las dos variables más importantes son el tiempo de residencia en la ciudad y la tipología de estudios realizados. La importancia del tiempo de residencia se explica por tener un mejor nivel de inglés y por estar más integrado en la sociedad y conocer mejor el mercado laboral del país. No obstante, el tiempo de residencia en Londres no es tan importante cuando se ha estudiado una carrera muy demandada en el país. Hemos visto que las profesionales de la salud consiguen un trabajo calificado mucho antes que los titulados en ciencias sociales o en artes; mientras que los ingenieros tienen una inserción laboral intermedia, más rápida que los titulados en ciencias sociales pero no tan inmediata como las profesionales de la salud. En el artículo también se han dibujado diferentes trayectorias para entrar en el mercado de trabajo de Londres. Aparte de las experiencias en la búsqueda de empleo en la capital británica también se han comentado otras iniciativas laborales surgidas a partir de la emigración española en Londres, como las agencias que ofrecen vivienda y trabajo a los jóvenes que emigran por primera vez a Londres y que buscan hacerlo con el menor riesgo posible.

La revisión de la bibliografía y los datos recogidos en el trabajo de campo nos permite afirmar que no todos los jóvenes emigran exclusivamente por ausencia de oportunidades de trabajo en su país de origen. Algunos de ellos deciden hacerlo por motivos de estudio o para satisfacer una inquietud personal. De cualquier modo, los jóvenes entrevistados manifiestan que esta emigración les permitirá mejorar 
sus competencias y tener más oportunidades laborales al volver a su país de origen, principalmente por el hecho de tener un nivel superior de inglés o por haber tenido experiencias laborales en el extranjero.

Como se ha podido ver en el presente estudio, la opción de aceptar trabajos no calificados (aproximación sucesiva) supone solo una de las varias opciones que los jóvenes toman en vías a conseguir un trabajo calificado. No obstante, se trata de la condición más común entre los y las jóvenes entrevistados, en especial entre aquellos recién llegados.

No obstante, esto puede llegar a ser una paradoja. Estas personas jóvenes dejan su país de origen porque solo les ofrece trabajos no calificados y precarios (incluso aquellas que se van sin haber buscado trabajo anteriormente, se marchan con la idea que es imposible conseguir un trabajo calificado en España), y se van a otro país para encontrar uno mejor, aunque en algunas ocasiones acaben aceptando las mismas condiciones precarias que ofrece el mercado laboral español, y en algunos casos peores. ¿Por qué? Este estudio apunta un elemento que puede ser aclarativo. Muchos de los y las jóvenes entrevistados se sienten inseguros al llegar a la ciudad, sobre todo por su bajo nivel de inglés, pero también por el hecho de encontrarse en un contexto totalmente nuevo, lo cual puede suponer una barrera para acceder al mercado de trabajo calificado. En ese sentido, delante de la necesidad de pagar las facturas, acceden a tomar trabajos no calificados porque es la manera más sencilla y rápida de entrar en el mercado laboral, siendo esta una situación temporal condicionada a hallar un trabajo relacionado con los estudios, aunque esta condición no siempre se cumpla.

Desde un punto de vista antropológico este fenómeno también se podría explicar a través de las expectativas. Los jóvenes piensan en todo momento que la situación de precariedad laboral cambiará en un futuro y conseguirán el trabajo que deseaban. En el imaginario colectivo español se relaciona la migración con una necesidad de empezar "desde abajo"; de acuerdo con este imaginario, un inmigrante debe empezar con trabajos no calificados, teniendo siempre la opción de ir ocupando puestos mejores cuando su integración a la sociedad de acogida sea mayor. Desde nuestro análisis consideramos que estas expectativas juegan un papel muy importante en las mentes de las personas jóvenes emigradas a Londres. No obstante, este fenómeno abre nuevas preguntas, por ejemplo, por qué una persona joven calificada se queda desarrollando permanententemente trabajos no calificados en Londres sin atreverse a buscar el trabajo ideal que se fue a buscar, como es el caso de algunos de los informantes que después de varios meses haciendo trabajos no cualificados aún no habían dado el paso a trabajos cualificados acorde con sus estudios. 


\section{Referencias}

Alberich, Neus; Clara Rubio, Pau Serracant (2016), Marxar per tornar. L'adquisició de competències professionals en l'emigració internacional de persones joves, Barcelona, Aportacions, 56 .

Barbagelata, Héctor-Hugo (2012), "Youth, unemployment and education", en Alfredo Sánchez-Castañeda, Lavinia Serrani y Francesca Sperotti (eds.), Youth Unemployment and joblessness: causes, consequences, responses, Cambridge: Cambridge Scholars, pp. 3-II.

Barómetro del Real Instituto Elcano (BRIE) (2OI3), 32 a Oleada BRIE, febrero 2013. Disponible en: <http://www.realinstitutoelcano.org/wps/portal/ rielcano_es/encuesta?WCM_GLOBAL_CONTEXT=/elcano/elcano_es/ barometro/oleadabrie32> [consulta: 23/05/2018].Bessant, Judit; Rys Farthing, Rob Watts (2017), The Precarious Generation: A Political Economy of Young People. Londres, Routledge.

Castles, Stephen y Mark Miller (1966), The age of migration, Londres, McMillan Press.

Centro de Investigaciones Sociológicas (CIS) (2012), Barómetro de Febrero. Disponible en: <http://datos.cis.es/pdf/Es2932mar_A.pdf > [consulta: 05/04/2017].

Domingo, Andreu y Amand Blanes (2015), "Inmigración y emigración en España: estado de la cuestión y perspectivas de futuro", еn сіров. Anuario de la Inmigración en España 2014, Barcelona, СІров publicacions, pp. 94-II2. Domingo, Andreu y Albert Sabater (2013), "Crisis económica e inmigración: la perspectiva demográfica”, en: E. Aja, J. Arango y J. Oliver (dirs.), Inmigración y crisis: entre la continuidad y el cambio, Barcelona, сгоов publicacions, pp. 6I-87.

Faist, Thomas y Eyüp Ozveren (eds.) (2004), Transnational Social Spaces: agents, networks and institutions, Aldershot, Ashgate.

Fashoyin, Tayo (2012), "Foreward" en Alfredo Sánchez-Castañeda, Lavinia Serrani y Francesca Sperotti (eds.), Youth unemployment and joblessness: causes, consequences, responses, Cambridge, Cambridge Scholars, pp. XV-XVII.

Favell, Adrian, Miriam Feldblum y Michael Peter Smith (2006), "The human face of global mobility: a research agenda”, en: Michael Peter Smith y Adrian Favell (eds.), The human face of Global Mobility: International 
bighly skilled migration in Europe, North America and the Asia-Pacific, Londres, Transaction Publishers, pp. I-26.

Feixa, Carles; Clara Rubio (2017),"Introducción: Te vas pensando que has dejado atrás a zombies. La emigración juvenil: ¿aventura o exilio?" Revista de Dialectología y Tradiciones Populares, 72(I), pp. 9-22.

Fundación CIPD (2013), The State of Migration: employing migrant workers. Disponible en: <http://www.cipd.co.uk/publicpolicy/policy-reports/ employing-migrant-workers.aspx $>$ [consulta: 05/04/2017]

González-Ferrer, Amparo (2013),"La nueva emigración española. Lo que sabemos y lo que no", Laboratorio de Alternativas, i8.

Hatton, Timothy J. y Jeffrey G. Williamson (1998), The Age of Mass Migration. Causes and Economic Impact, Oxford, Oxford University Press.

Hooghe, Marc, Ann Trappers, Bart Meuleman y Tim Reeskens (2008), "Migration to European Countries: A Structural Explanation of Patterns, I980-2004". IMR, 42(2), pp. 476-504.

Jiménez, Cecilia (20II), “¿Empobrecimiento o desclasamiento? La dimensión simbólica de la desigualdad social" Trabajo y Sociedad, 15(I7)

Kalter, Frank (1998), "Partnerschaft und Migration. Zur Theoretischen Erklärung Eines Empirschen Effect”, Kölner Zeitschrift fu凶r Soziologie und Sozialpsychologie 5o(2), pp. 283-309.

Kuptsch, Christiane y Eng Fong Pang (2006), Competing for global talent, Ginebra, International Labour Office.

Petras, James (1995), El informe Petras. CGT Publicaciones. Disponible en línea; $<$ https://www.inventati.org/ingobernables/textos/anarquistas/informe-petras.pdf $>$ [consulta: 23/05/2018].Portes, Alejandro y Kelly Hoffman (2003), “La estructura de clases en América Latina: composición y cambios durante la era Neoliberal", Desarrollo económico, 43(I7I).

Rubio, Clara y Tanja Strecker (2016), "Young Catalans living in London: 'You can make lots of money here, but it's a very hostile city as well". Youth Voice Journal. Disponible en línea: <https://youthvoicejournal. com/2016/12/12/clara-rubio-2016young-catalans-living-in-london-you -can-make-lots-of-money-here-but-its-a-very-hostile-city-as-well/> [consulta: 05/04/2017].

Rodríguez- Puertas, Rubén y Francisco Entrena-Durán (2017), "Un estudio comparativo de los procesos de adaptación en jóvenes españoles emigrados al Reino Unido y a Chile durante el período 20IO-2014", EMPIRIA. Revista de Metodología de Ciencias Sociales, 37, pp. 4I-73. 
Rubio, Clara y Tanja Strecker (2017), "Aquí i allà: els plans d'emigració de les persones joves universitàries i les realitats que troben a Londres", en: Carles Feixa, Clara Rubio, Joan Ganau, Francesc Solsona (eds.), L'Emigrant 2.o. Emigració juvenil, moviments socials i xarxes digitals, Barcelona, Observatori Català de la Joventut.

Rubio, Clara (2017), L'emigració dels joves catalans a Londres. Precarietat juvenil, mobilitat exterior i moviments socials, tesis doctoral inédita, Universitat de Lleida.

Salvadó, Toni (2009), "Joves i mercat de treball. Malestar juvenil o malestar generacional?", Nous Horitzons, I89, pp. 19-26.

Scarpetta, Stefano, Anne Sonnet y Thomas Manfredi (2010), "Rising Youth Unemployment During The Crisis: How to Prevent Negative Longterm Consequences on a Generation?", en OECD Social, Employment and Migration, Working Papers, 106.

Serracant, Pau (2005), "La sobrequalificació entre els joves catalans", Col.lecció Aportacions, 27.

Walther, Andreas (2006), "Regimes of youth transitions. Choice, flexibility and security in Young people's experiences across different European contexts", Young, I4(29), pp. II9-139.

Clara Rubio Ros

Doctora en Antropología por la Universidad de Lleida. Tiene una licenciatura en Pedagogía y otra en Antropología Social y Cultural (ambas a la Universidad Autónoma de Barcelona), además tiene un Máster interuniversitario en Juventud y Sociedad por la Universidad de Girona. Ha realizado estancias doctorales en la Open University y en el Royal Anthropological Institute, ambas en el Reino Unido. Entre sus publicaciones más recientes destacan: "El tratamiento mediático de la emigración juvenil en los informativos televisivos y en los videos autoproducidos por migrantes (2009-2015)" en la Revista Latina de Comunicación Social; "Young Catalans living in London: 'You can make lots of money here, but it's a very hostile city as well”, Youth Voice Journal y el libro Emigrant 2.0. Emigració juvenil, nous moviments socials $i$ xarxes digitals. 
Citar como: Rubio, Clara (2018),"Jóvenes catalanes en Londres: emigración juvenil, crisis y mercado de trabajo", Iztapalapa. Revista de Ciencias Sociales y Humanidades, núm. 85, año 39, julio-diciembre de 2018, ISSN: 2007-9176; pp. 169-194. Disponible en <http://revistaiztapalapa.izt.uam.mx/index.php/ izt/issue/archive $>$. 